\title{
Spatially-resolved Observation of the Excitation Temperature in a Glow Discharge Plasma for Atomic Emission Spectrometry
}

\author{
Munehiko MatsuURA and Kazuaki Wagatsuma ${ }^{\dagger}$ \\ Institute for Materials Research, Tohoku University, 2-1-1 Katahira, Sendai 980-8577, Japan
}

\begin{abstract}
A two-dimensionally imaging spectrometer system was employed to obtain the spatial distribution of the excitation temperature in a glow discharge plasma for emission analysis. A Grimm-style glow discharge excitation source, whose hollow anode had an inner diameter of $8 \mathrm{~mm}$, was observed over the whole emission area. The mapping of the excitation temperature was conducted by using a two-line method under the assumption of the Boltzmann distribution. Iron was chosen as the probe element, and a pair of the atomic lines: Fe I $371.99 \mathrm{~nm}(3.33 \mathrm{eV})$ and Fe I $375.82 \mathrm{~nm}(4.25 \mathrm{eV})$, were employed for the measurement. The excitation temperature was a little varied over the plasma region except for the edge portion, even when the discharge voltage as well as the argon pressure were changed as the experimental parameters. The temperature was estimated to be $4650-4950 \mathrm{~K}$ at a central portion of the plasma having a 5-mm-diameter circle, implying that the emission excitation at the central portion occurred almost uniformly for each discharge condition.
\end{abstract}

(Received December 10, 2010; Accepted January 23, 2011; Published March 10, 2011)

\section{Introduction}

In glow discharge optical emission spectrometry (GD-OES), the plasma is self-sustained to emit the characteristic radiation with small variations as well as with low background levels, yielding not only large signal-to-noise but signal-to-background ratios; therefore, it is essentially suitable for elemental analysis requiring good precision and repeatability. ${ }^{1}$ GD-OES is also employed for the direct analysis of solid samples, because sample atoms are directly introduced into the glow discharge plasma through cathode sputtering. ${ }^{2}$ The sputtering process enables GD-OES to be a rapid analytical method for obtaining a depth profile of the sample composition, because it requires only little sample pretreatment without ultra-high vacuum conditions. $^{3-7} \quad$ In a conventional measurement system for GD-OES, the emission signal is observed from the axial direction of the plasma when it is collected onto an entrance slit of the spectrometer with a point-focused lens. Therefore, the intensity of an emission line is integrated over a certain area of the plasma, enabling it to be estimated with better precision; however, it cannot give information about the spatial distribution of the emission intensity at different portions of the plasma.

A dispersed image of the glow discharge plasma can be obtained with a particular spectrometer system, where the emitted radiation is directly collimated on the grating of the spectrometer and the plasma image after dispersion is projected on a two-dimensional (2D) detector. However, a few papers have been reported on the 2D spectral image in GD-OES, principally because of a lack of spectrometers enabling 2D observation having a good spectral resolution. Gamez et al. first conducted spatially-resolved elemental analysis in GD-OES by using an imaging spectrometer system with a pulsed

$\dagger$ To whom correspondence should be addressed.

E-mail:wagatuma@imr.tohoku.ac.jp radio-frequency power supply, indicating that small particles embedded in an organic sample could be separately detected in the elemental mapping. ${ }^{8}$ We have been interested in spatial variations in the emission characteristics of a glow discharge plasma. Our previous papers reported on 2D spectral images of zinc and copper samples by using a two-dimensionally imaging spectrometer having a spectral resolution of less than $1 \mathrm{~nm}$, indicating that the intensities of their emission lines were not uniform over the plasma area, but were drastically reduced along the radial direction of the plasma. ${ }^{9,10}$ We were very interested in the reason why such a spatial distribution of the emission intensity was caused, in order to clarify the excitation mechanism occurring in the glow discharge plasma. Two possible reasons for this would be considered as follows: (1) the excitation efficiency is inhomogeneous in the plasma and (2) the number density of analyte species is inhomogeneous in the plasma. For this research, it is useful to measure a 2D image of the excitation temperature in the plasma, which has not been reported in previous papers. In the present work, the two-dimensional/spatial distribution of the excitation temperature was estimated by using a two-line method between iron atomic lines.

\section{Experimental}

\section{Apparatus}

A glow discharge excitation source was made in our laboratory ${ }^{11}$ according to an original model of Grimm, ${ }^{12,13}$ where the inner diameter of the hollow anode was $8.0 \mathrm{~mm}$ and the distance between the anode and cathode sample was adjusted to be $0.2-0.4 \mathrm{~mm}$. The emitted radiation was observed from the axial direction of the plasma, and it looked like that the plasma was uniformly bright within the hollow anode. The imaging spectrometer system comprised a collimator optics, an image spectrograph, and a charge-coupled device (CCD) detector. ${ }^{9,14}$ 
The emission signal from the excitation source was introduced through the collimator onto the entrance slit of the spectrograph (Model 12580, BunkoKeiki Corp., Japan), and then the emission image dispersed at a certain wavelength was detected on the CCD detector (SensiCam QE Model, PCO Imaging Corp., Germany), where the 2D image of a particular emission line could be observed in the radial direction of the plasma. The optical alignment between the glow discharge excitation source and the spectrometer was adjusted by using zero-order diffraction light, so that an image of the source could be observed the most clearly. The Grimm-style excitation source produces an obstructed glow discharge plasma, where the emission zone, called a negative glow, is localized just above the sample surface having a thickness as thin as the mean free path of sputtered sample atoms., ${ }^{2,15}$ It was thus considered that the emission image was obtained from the negative glow zone, itself. It was determined by measuring an image of a scale that a 2D image obtained by $10 \times 10$ pixels approximately corresponded to an actual sample area of $0.21 \times 0.21 \mathrm{~mm}^{2}$. The spectral resolution was $0.1-1.0 \mathrm{~nm}$, depending on the slit width. The data were accumulated and averaged on a personal computer to improve the spatial resolution and the plasma image was finally recorded. A dc power supply (Model HV1.5-1, Takasago Ltd., Japan) was employed so that the discharge voltage could be varied at several pressures of the plasma gas.

High-purity argon (>99.999\%) was introduced as the plasma gas after evacuating the chamber to below $10 \mathrm{~Pa}$. A Pirani vacuum gauge (GP-2, ULVAC Corp., Japan), which had been corrected for pure argon, was placed between the evacuation port and a rotary vacuum pump (GLD-166, ULVAC Engineering Inc., Japan). The plasma gas was flowed during the measurement while keeping a pre-determined chamber pressure. A pure iron plate $(99.9 \%$, purity) was prepared as the sample. The sample surface was cleaned with acetone, and then fixed at the sample port of the chamber.

\section{Two-line method}

A characteristic temperature can be defined under the Boltzmann distribution. In emission measurements, an excitation temperature is determined for a pair of spectral lines having different excitation energies, if their transition probabilities are known. This method is based on the difference in the number density between two energy levels, which can be determined by a characteristic temperature under thermodynamic equilibrium. ${ }^{16}$ The general equation for the excitation temperature $(T)$ is denoted as follows: ${ }^{16}$

$$
T=\frac{5040\left(E_{\mathrm{a}}-E_{\mathrm{b}}\right)}{\log \left[(g A)_{\mathrm{a}} /(g A)_{\mathrm{b}}\right]-\log \left(\lambda_{\mathrm{a}} / \lambda_{\mathrm{b}}\right)-\log \left(I_{\mathrm{a}} / I_{\mathrm{b}}\right)}
$$

where $E$ means the excitation energy in $\mathrm{eV}, g A$ is the transition probability, $\lambda$ is the wavelength, and $I$ is the intensity when two different spectral lines $(a, b)$ are measured.

In this study, a pair of iron atomic lines, Fe I $371.99 \mathrm{~nm}$ and Fe I $375.82 \mathrm{~nm}$, was employed as probe lines for the temperature measurement. The reason for this line selection is that these $\mathrm{Fe} \mathrm{I}$ lines are in an adjacent wavelength region, in which the sensitivity of the spectrometer is almost the same, and that they are not overlapped with neighboring emission lines. The former line is assigned to the transition from the $3 \mathrm{~d}^{6} 4 \mathrm{~s} 4 \mathrm{p}{ }^{5} \mathbf{F}_{5}(3.33 \mathrm{eV})$ to the $3 \mathrm{~d}^{6} 4 \mathrm{~s}^{2}{ }^{5} \mathbf{D}_{4}(0.00 \mathrm{eV})$ levels having a $g A$ value of $1.739 \times 10^{8} \mathrm{~s}^{-1}$, and the latter line to the transition from the $3 \mathrm{~d}^{6} 4 \mathrm{~s} 4 \mathrm{p}{ }^{5} \mathbf{F}_{3}(4.26 \mathrm{eV})$ to the $3 \mathrm{~d}^{6} 4 \mathrm{~s}^{2}{ }^{5} \mathbf{F}_{3}(0.96 \mathrm{eV})$ levels having a $g A$ value of $4.438 \times 10^{8} \mathrm{~s}^{-1} .{ }^{17}$ The energy difference between the $3 d^{6} 4 \mathrm{~s} 4 \mathrm{p}{ }^{5} \mathbf{F}_{5}$ and the $3 d^{6} 4 \mathrm{~s} 4 \mathrm{p}^{5} \mathbf{F}_{3}$ levels is the most important

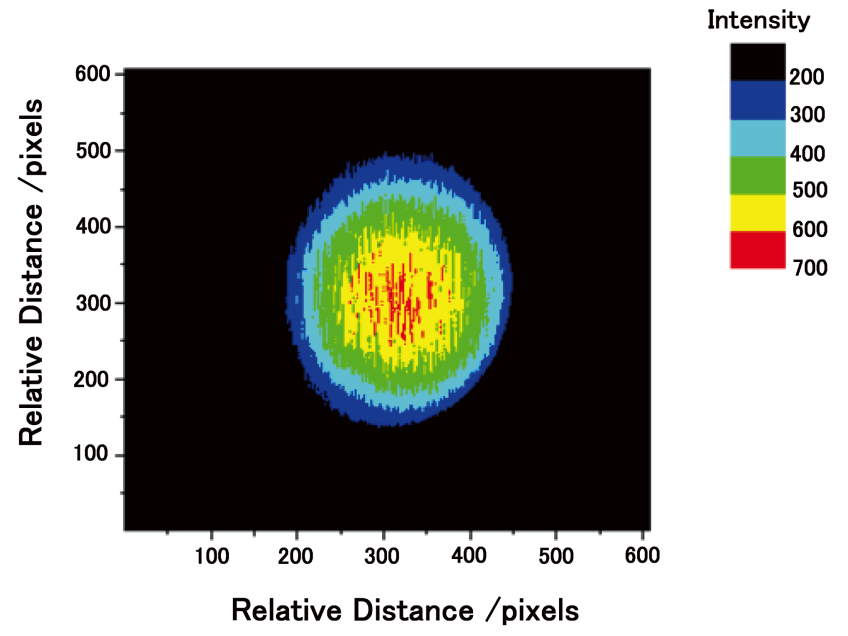

Fig. 1 Two-dimensional emission image of the Fe I 371.99-nm line observed from the axial direction of a glow discharge plasma. Sample, pure iron; discharge voltage, $600 \mathrm{~V}$; Ar pressure, $800 \mathrm{~Pa}$; gate width of the CCD detector, $1 \mathrm{~ms}$; the number of data accumulation, 900.

factor in determining the excitation temperature.

After substituting these parameters in Eq. (1), a series of the excitation temperature is calculated from the intensity ratio for each pixel of the $\mathrm{CCD}$ detector corresponding to different portions of the plasma. A spatial distribution of the excitation temperature in the plasma can be converted from such two-dimensional matrix of the intensity ratio.

\section{Results and Discussion}

Image of the emission intensity

Our previous papers have already represented 2D emission images of the glow discharge plasma for pure zinc, pure copper, and zinc-coated steel samples, indicating that there is a common feature among them which is less dependent on the sample materials, the analytical emission lines and the discharge conditions. ${ }^{9}, 10$ They demonstrated that emissions of the samples were not irradiated uniformly over the possible plasma zone, but had a large variation in the intensity along the radial distance. The central portion, having a diameter of about $2 \mathrm{~mm}$, gave the most intense emission, and the intensity was drastically reduced towards the radial direction of the plasma. ${ }^{9}$

Figure 1 shows a 2D emission image for the Fe I 371.99-nm line in a pure iron sample, whose intensities are expressed by mapping with several colors, when the glow discharge plasma is maintained at a voltage of $600 \mathrm{~V}$ and an argon pressure of $800 \mathrm{~Pa}$. In this case, the emission intensity was varied from 200 to 700 in an arbitrary unit, where the lowest value was comparable to the background level. The slit width of the spectrometer was set to be $2.0 \mathrm{~mm}$. The gate width of the CCD detector was $1 \mathrm{~ms}$ and sampling of the signal was carried out at a frequency of $1 \mathrm{kHz}$. The emission signal was stored in the CCD detector during 32 timing pulses, and this accumulation was 900-times replicated and averaged on a personal computer. In this image, 100 pixels corresponded to $2.12 \mathrm{~mm}$ in the actual plasma size. The emission image of the Fe I line has a similar feature to the images of the $\mathrm{Zn} \mathrm{I}$ and the $\mathrm{Cu}$ I lines, which were indicated in our previous research: the concentric-circle pattern means that the emission intensity becomes drastically reduced from the central part to the edge portions of the plasma. 


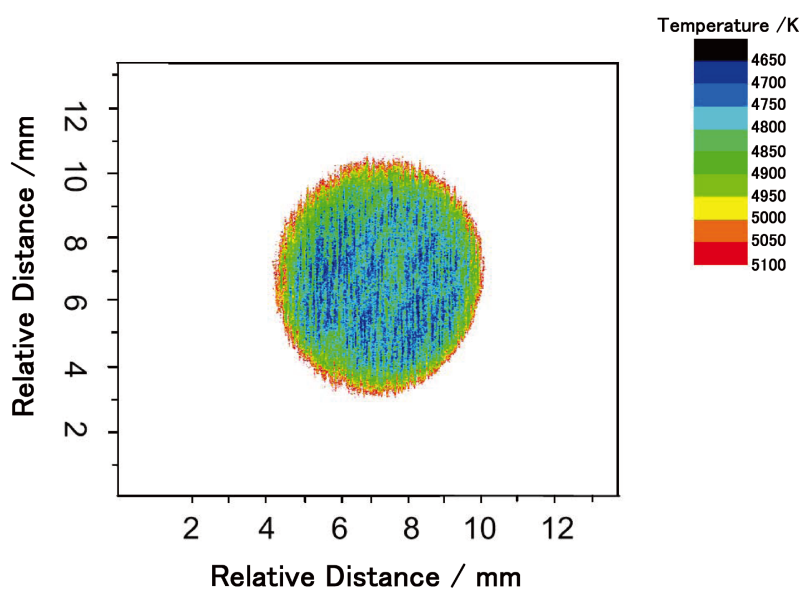

Fig. 2 Two-dimensional map for the excitation temperature estimated with a two-line method between Fe I $371.99 \mathrm{~nm}$ and Fe I $375.82 \mathrm{~nm}$. The discharge conditions and the measuring conditions are the same as in Fig. 1.

This effect would be because any plasma parameters determining the excitation processes, such as the excitation temperature, are changed in the radial direction of the glow discharge plasma, or it is another probable reason for this effect that the number density of the analytes, such as iron atoms, becomes larger at the central portion of the plasma. In order to investigate the cause of the inhomogeneous intensities in the plasma, we measured the 2D spatial distribution of the excitation temperature by using a two-line method between the Fe I lines.

\section{$2 D$ image of the excitation temperature}

Figure 2 shows a typical 2D distribution of the excitation temperature obtained by the two-line method, which is expressed with several colors in the temperature range of $4650-5050 \mathrm{~K}$, when the glow discharge plasma is maintained at a voltage of $600 \mathrm{~V}$ and an argon pressure of $800 \mathrm{~Pa}$. The measuring conditions were the same as those in Fig. 1. The 2D image became slightly enlarged in the vertical direction, which might have been caused by any aberration through the slit of the spectrograph; however, the variation in the excitation temperature could be clearly found. It was little changed in the radial direction but was distributed almost uniformly over the central portion having a diameter of $4-5 \mathrm{~mm}$, although irregular fluctuations in the excitation temperature were included in the data points. The outermost surrounding region seemed to have higher temperatures than the central area; however, this was mainly derived from a larger uncertainty in the excitation temperature because of the very faint emission intensities. Therefore, the surrounding region should not be included in the following discussions. Figure 3 shows plots of the mean excitation temperature, which are averaged over each area of $10 \times 10$ pixels, along the radial direction of the plasma, indicating that the excitation temperature is maintained almost constant in the central portion of the plasma. In this case, the average excitation temperature over the data points was estimated to be $4770 \mathrm{~K}$.

It is clear to say that the spatial distribution of the excitation temperature shown in Fig. 2 cannot explain that of the emission intensity (see Fig. 1); the emission intensity is more reduced with increasing radial distance, although little variations in the excitation temperature is found over a relatively wide portion of the plasma. This result implies that the concentric-circle-like

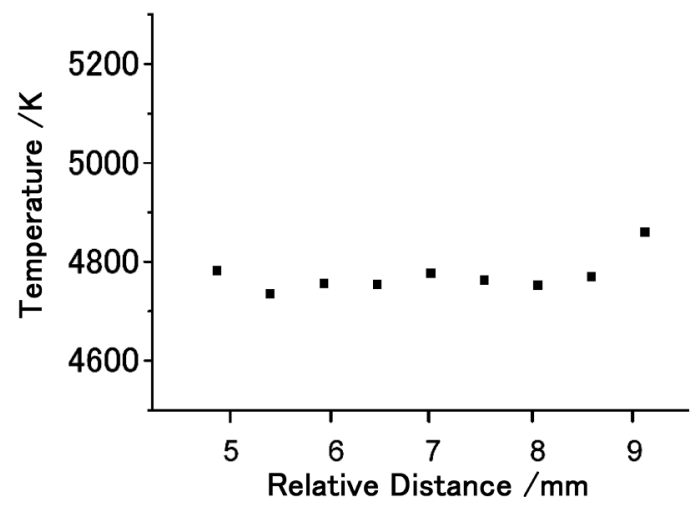

Fig. 3 Plots of the average excitation temperature which is averaged over $10 \times 10$ pixels in the radial direction of the plasma. The discharge conditions and the measuring conditions are the same as in Fig. 1.

variation in the emission intensity is not derived from any excitation process in the plasma, but from the number density of iron atoms decreasing towards the edge portion of the plasma.

Figure 4 shows a comparison in 2D images of the excitation temperature at several argon pressures of 530, 670, and $800 \mathrm{~Pa}$, when the discharge voltage was fixed at $600 \mathrm{~V}$. The measuring conditions were the same as those for Fig. 1. The average excitation temperature, which was estimated over the central portion of the plasma having a diameter of $c a .5 \mathrm{~mm}$, was $4920 \mathrm{~K}$ at $530 \mathrm{~Pa}, 4780 \mathrm{~K}$ at $670 \mathrm{~Pa}$, and $4740 \mathrm{~K}$ at $800-\mathrm{Pa} \mathrm{Ar}$, which slightly decreased with the argon pressure. However, when the argon pressure was varied, the spatial distributions in the excitation temperature were not varied largely: they were almost uniform over the 5-mm-diameter area of the plasma. Figure 5 shows a comparison in 2D images of the excitation temperature, when the discharge voltage was set to be 600, 700, and $800 \mathrm{~V}$ at an argon pressure of $530 \mathrm{~Pa}$. The measuring conditions were the same as in Fig. 1. The average excitation temperature, which was estimated over the central portion of the plasma having a diameter of $c a .5 \mathrm{~mm}$, was $4920 \mathrm{~K}$ at $600 \mathrm{~V}$, $4780 \mathrm{~K}$ at $700 \mathrm{~V}$, and $4690 \mathrm{~K}$ at $800 \mathrm{~V}$; therefore, it was gradually reduced with increasing discharge voltages. As similar to the argon-pressure dependence shown in Fig. 4, the spatial distribution in the excitation temperature had a similar pattern, regardless of the applied discharge voltage. It is therefore considered that the $2 \mathrm{D} /$ spatial distribution has a common feature being less dependent on the discharge conditions for producing the plasma: for each discharge condition, the emission excitation occurs almost uniformly at the central portion of the plasma, even if the average values of the excitation temperature are slightly changed by the discharge parameters.

Based on the 2D images of the excitation temperature, as shown in Figs. $2-5$, we could suggest that a large spatial variation in the emission intensity (see Fig. 1) does not result from the excitation efficiency, but from a corresponding variation in the number density of iron atoms in the radial direction of the plasma. A probable reason for this is that a flow of the plasma gas would determine the residual time of sputtered iron species and eventually the distribution of the number density in the plasma. Figure 6 schematically illustrates the evacuation system for introducing the plasma gas into the glow discharge excitation source employed in this study. It comprised two rotary vacuum pumps: one evacuated the discharge chamber and the other worked as an auxiliary vacuum pump to keep the 

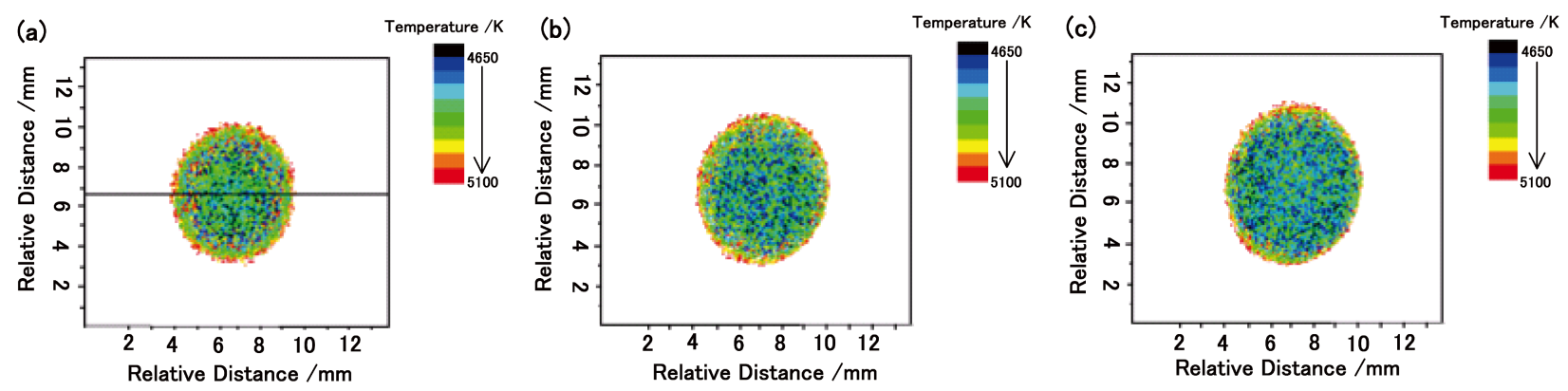

Fig. 4 Two-dimensional maps for the excitation temperature at argon pressures of $530 \mathrm{~Pa}$ (a), $670 \mathrm{~Pa}$ (b), and $800 \mathrm{~Pa}(\mathrm{c})$, when the discharge voltage was fixed to be $600 \mathrm{~V}$.
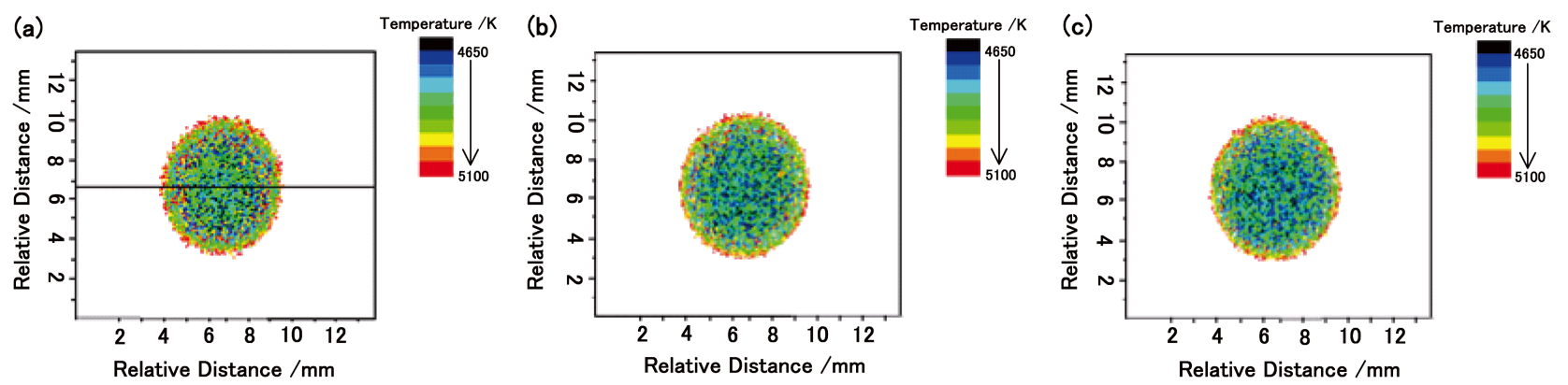

Fig. 5 Two-dimensional maps for the excitation temperature at discharge voltages of $600 \mathrm{~V}$ (a), $700 \mathrm{~V}$ (b), and $800 \mathrm{~V}$ (c), when the argon pressure was fixed to be $530 \mathrm{~Pa}$.

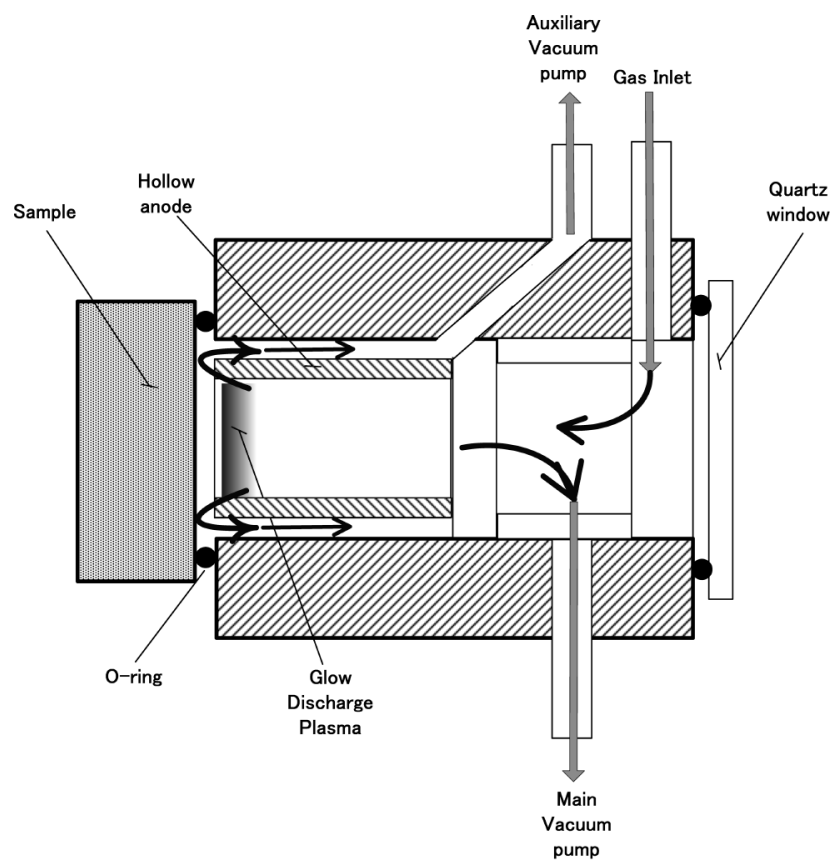

Fig. 6 Schematic diagram of the glow discharge excitation source for representing the flow of the plasma gas.

vacuum at a sealing portion (o-ring) for the sample, so as to obtain a stable discharge condition. This vacuum path, which was conducted from the outside of the hollow anode (see Fig. 6), was able to lead to a faster flow of the plasma gas containing the sputtered iron species, and thus the decreased density of iron atoms at the edge portion of the plasma. This effect would cause smaller emission intensities at the surrounding portion compared to the central portion of the plasma, and the concentric-circles pattern in the emission intensity could be reasonably explained by a circle-symmetric flow of the plasma gas at the outside portion of the hollow anode.

\section{Conclusions}

Two-dimensional emission images for iron atomic lines in a pure iron sample were observed to investigate any dynamic variation in the excitation characteristics occurring in a glow discharge plasma. The emission image was not uniform in the radial direction of the plasma, but had a large variation in the intensities along the radial distance. This phenomenon was similar to two-dimensional emission images of other elements that had been reported in our previous papers. In order to clarify the reason for this effect, we further investigated the spatial distribution of the excitation temperature along the radial direction of the plasma, which was estimated with a two-line method using iron atomic lines. The two-dimensional measurements indicated that the plasma had a central zone (the diameter of $c a .5 \mathrm{~mm}$ ) where the excitation temperature was a little changed, and such distribution was commonly observed under various discharge conditions, where the argon gas pressure as well as the discharge voltage was varied. Therefore, it can be suggested that the intensity variations at different plasma zones are probably because iron atoms have a larger number density at the central portion of the plasma after sputtering, due to the differential pumping system in the excitation source. 


\section{Acknowledgements}

The authors gratefully acknowledge financial support by a grant from the Ministry of Environment, Japan. This research is supported also by Grant-in-Aids from the Ministry of Education, Science, Sports and Culture of Japan (No. 18360016).

\section{References}

1. R. Payling, D. G. Jones, and A. Bengtson (ed.), "Glow Discharge Optical Emission Spectrometry", 1997, John Wiley \& Sons, Chichester.

2. R. Mavrodineau, J. Res. Natl. Bur. Stand., 1984, 89, 143.

3. J. Pons-Corbeau, J. P. Cazet, J. P. Moreau, R. Berneron, and J. C. Charbonnier, Surf. Interface Anal., 1986, 9, 21.

4. S. Suzuki and K. Suzuki, Surf. Interface Anal., 1991, 17, 551.

5. A. Bengtson and M. Lundholm, J. Anal. At. Spectrom.
1988, 3, 879 .

6. R. Payling and D. G. Jones, Surf. Interface Anal., 1993, 20, 787.

7. H. Park and K. Wagatsuma, Anal. Sci., 2006, 22, 599.

8. G. Gamez, S. J. Ray, F. J. Andrade, M. R. Webb, and G. M. Hieftje, Anal. Chem., 2007, 79, 1317.

9. Y. Zenitani and K. Wagatsuma, Anal. Sci., 2008, 24, 555.

10. Y. Zenitani, F. Sakamoto, and K. Wagatsuma, Anal. Sci., 2009, 25, 323 .

11. K. Wagatsuma and K. Hirokawa, Surf. Interface Anal., 1986, 8, 37.

12. W. Grimm, Naturwissenschaften, 1967, 54, 586.

13. W. Grimm, Spectrochim. Acta, Part B, 1968, 23, 443.

14. C. Kitaoka and K. Wagatsuma, Anal. Sci., 2007, 23, 1261.

15. A. von Engel, "Ionized Gases", 1965, Clarendon Press, Oxford.

16. P. W. J. M. Boumans, "Theory of Spectrochemical Excitations", 1966, Plenum Press, New York.

17. J. R. Fuhr, G. A. Martin, and W. L. Wiese, J. Phys. Chem. Ref. Data, 1988, 17(Suppl.), No. 4. 